\title{
OBSERVATION OF ARGON LINES AT NORMAL PRESSURE IN THE VACUUM ULTRAVIOLET
}

\author{
D. MÜLLER, Ć. VADLA, and V. VUJNOVIĆ \\ Institute of Physics of the University Zagreb, Yugoslavia
}

A normal pressure arc having local thermal equilibrium, is a useful source for measurements of atomic parameters in the vacuum ultraviolet region (Boldt, 1970). However, it imposes severe difficulties if the spectral lines ending on low-lying levels should be observed. The reason is the absorption effective in cold and inhomogeneous gas layers intervening in the optical path between the homogeneous hot plasma column and the vacuum monochromator. Here a version of a cascade arc with a differential pumping system is described which makes possible the observation of argon resonance lines at 1067 and $1048 \AA$, considerably free of absorption. For this purpose it was found necessary to use a bored anode as a pumping port. In Figure 1 the anode part

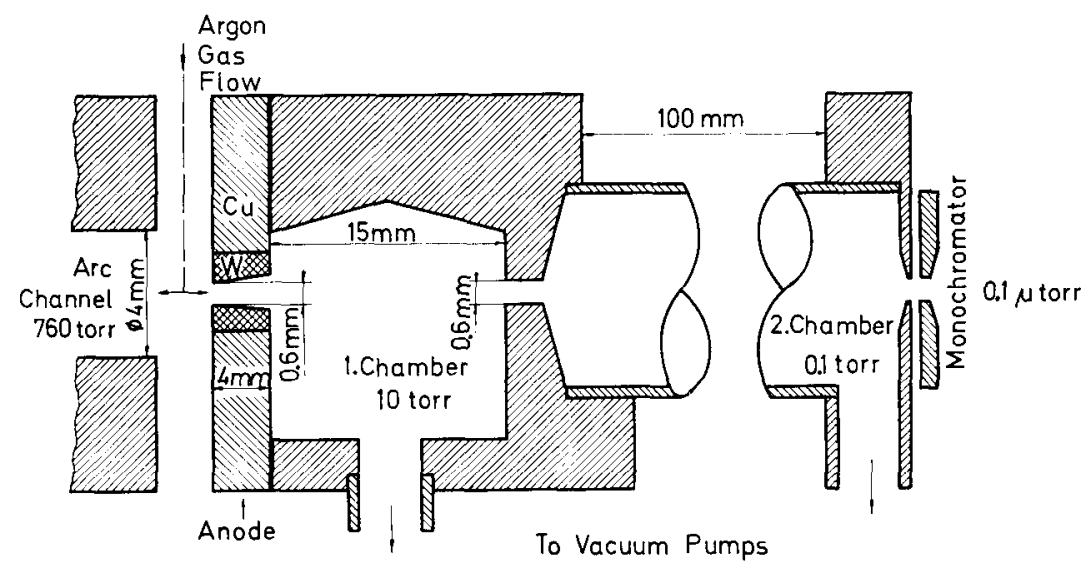

Fig. 1.

of the arc chamber is shown together with the differential pumping system (not to scale). The first pump (rotary, single stage, $30 \mathrm{~m}^{3} / \mathrm{h}^{-1}$ ) evacuates hot gases through the bored tungsten anode and reduces the pressure from atmospheric to 10 Torr. Inhomogeneous, cold gas layers exist only inside the anode bore and are strongly diluted in the optical path of the first and second pumping chambers.

The length of the first chamber is critical. If the length is too small, the gas jet hits the next diaphragm and the pumping is not effective. (For greater lengths, the pumping is not any more effective but the absorbing layer is larger.) The second chamber was evacuated to 0.1 Torr with another pump (rotary, $12 \mathrm{~m}^{3} / \mathrm{h}^{-1}$ ). The diaphragms between the chambers serve for vacuum isolation and for optical align- 
ment since the observation is made end-on. The dimensions of the holes and the length of the second chamber are chosen according to the necessary resolution of the axial region of the arc, allowing for radial temperature distribution and light diffraction. For arc channels having a diameter larger than $3 \mathrm{~mm}$, the holes have an optimum diameter of 0.5 to $0.6 \mathrm{~mm}$.

The spectrum obtained is shown in Figure 2. The use of the anode as a pumping port gives the lowest possible layer of cold gas column between the arc plasma and



Fig. 2.

the monochromator, and therefore argon of only technical grade purity can be used. Another advantage of the device was found in a very low gas loss. With given diaphragms, the flow rate of gas introduced into the arc equals $40 \mathrm{~cm}^{3} \mathrm{~s}^{-1}$ when the discharge is extinguished. Owing to an increase of gas temperature and constancy of the pumped volume, with the discharge of $60 \mathrm{~A}$ (temperature of about $13000 \mathrm{~K}$ ), the consumption of the introduced cold gas decreases to $5 \mathrm{~cm}^{3} \mathrm{~s}^{-1}$. This makes it possible to use precious gases, such as helium.

\section{Reference}

Boldt, G.: 1970, Space Sci. Rev. 11, 728. 